\title{
Viewpoint
}

\section{Solution processed nanostructures towards efficient and low-cost water splitting}

\author{
Anna Fontcuberta i Morral \\ Laboratory of Semiconductor \\ Materials, Institute \\ of Materials, Eocle \\ Polytechnique Fédérale de \\ Lausanne, 1015 Lausanne, \\ Switzerland \\ E-mail: anna.fontcuberta- \\ morral@epfl.ch
}

This Viewpoint reports on the work by Iyengar et al entitlted 'Photoelectrochemical Performance of NiO-coated ZnO-CdS Core-Shell Photoanode' [1]. In the last few years, photoelectrochemistry has emerged as a realistic technology for energy storage $[2,3]$. In a photoelectrochemical cell (PEC), light is absorbed in a semiconductor, the electron-hole pairs separate and are used for a separate oxidation and reduction reactions at each the junction with the electrolyte. As a result, chemical fuels such as hydrogen, methanol and even more caloric fuels could be generated $[4,5]$. In the race for efficiency, the design of each electrode in the PEC device has turned out to be key [6]. Optimal design involves optimizing band alignment with the electrolyte, effective protection against corrosion and photonic design to enhance light absorption. In addition, a catalyst at the surface of the electrodes is needed so that the reactions do not become kinetically limited.

In their paper, Iyengar et al provide a smart and low-cost design of the photoanode of a PEC. They start by optimizing the conditions for the fabrication of a sparse array of $\mathrm{ZnO}$ nanorods that they then cover with $\mathrm{CdS}$. This layer provides the photonic design for high light absorption and at the same time ensures an effective electron-hole pair separation. On top of this structure, they then deposit a mesoporous layer of co-catalyst $\mathrm{NiO}$, formed by an intricate mesh of vertical nanosheets. Overall, this work exploits all advantages of nanostructures for photoelectrochemistry, ranging from the photonics to enhancing the kinetics of reactions and optimizing carrier collection. It is a beautiful work that sets the path towards higher efficiency in the hydrogen evolution reaction. This design scheme is universal, and could certainly be applied to other materials and photoelectrochemistry reactions. Last but not least, all materials are obtained by solution-processing methods and could therefore be easily scalable to larger device sizes and high mass volume production at a low cost. This paper should inspire other groups to go over this initial efficiency of $4 \%$ towards $10 \%$ or higher. The technological impact will then be major.

\section{References}

[1] Iyengar P, Das C and Balasubramaniam K R 2017 J. Phys. D: Appl. Phys. 50 10LT01

[2] Pinaud B A et al 2013 Energy Environ. Sci. 6 1983-2002

[3] Lewis N S and Nocera D G 2006 Proc. Natl Acad. Sci. 103 15729-35

[4] Walter M G, Warren E L, McKone J R, Boettcher S W, Mi Q X, Santori E A and Lewis N S 2010 Chem. Rev. 110 6446-73

[5] Rajeshwar K et al 2013 Chem. Phys. Chem. 14 2251-9

[6] Scheuermann A G, Lawrence J P, Kemp K W, Ito T, Walsh A, Chidsey C E D, Hurley P K and McIntyre P C 2016 Nat. Mater. 1599 